\title{
DNASEIL3 as a Prognostic Biomarker Associated with Immune Cell Infiltration in Cancer
}

This article was published in the following Dove Press journal:

OncoTargets and Therapy

\author{
Zenghua Deng ${ }^{1,2, *}$ \\ Mengmeng $\mathrm{Xiao}^{3,4, *}$ \\ Dexiao $\mathrm{Du}^{2}$ \\ Nan Luo ${ }^{1,2}$ \\ Dongfang Liu $^{2}$ \\ Tingting Liu $^{2}$ \\ Dongbo Lian ${ }^{1,2}$ \\ Jirun Peng ${ }^{1,2}$
}

'Ninth School of Clinical Medicine, Peking University, Beijing, 100038,

People's Republic of China; ${ }^{2}$ Department

of Surgery, Beijing Shijitan Hospital,

Capital Medical University, Beijing,

100038, People's Republic of China;

${ }^{3}$ Peking University International Hospital,

Beijing, 102206, People's Republic of

China; ${ }^{4}$ Eighth School of Clinical

Medicine, Peking University, Beijing,

102206, People's Republic of China

*These authors contributed equally to this work
Correspondence: Jirun Peng

Tel +86-10-6392672I

Email pengjr@bjsjth.cn
Objectives: Deoxyribonuclease 1 like 3 (DNASE1L3) is critically involved in apoptosis and immune response, however, its role in cancer has yet to be deciphered. We aimed to explore the prognostic value of DNASE1L3 across a series of malignancies.

Methods: Based on Oncomine database and Tumor Immune Estimation Resource (TIMER), expression profiling of DNASE1L3 was detailed in malignancies. Using PrognoScan, Kaplan-Meier Plotter, GEPIA2, and bc-GenEcMiner v4.5, prognostic value of DNASE1L3 was estimated in diverse cancers. Based on TIMER, association between DNASEL13 expression and immune infiltration was examined in various cancers. Then, mRNA level of DNASE1L3 in hepatocellular carcinoma (HCC) samples $(n=22)$ and stomach adenocarcinoma (STAD) samples $(n=17)$ was measured with qRT-PCR. Immunohistochemistry was performed to confirm expression of DNASE1L3 in paraffin-embedded tissues of HCC $(n=9)$ and lung adenocarcinoma $(\mathrm{n}=20)$.

Results: DNASE1L3 was downregulated in multiple cancers, including breast invasive carcinoma (BRCA), cholangiocarcinoma (CHOL), liver hepatocellular carcinoma (LIHC), and lung adenocarcinoma (LUAD). A lower level of DNASE1L3 correlated with poorer prognosis in various cancers, especially in breast, liver, kidney, stomach, lung adenocarcinoma and sarcoma (SARC). Moreover, DNASE1L3 was positively related to immune cell infiltration in many cancers, including BRCA, LIHC, STAD, LUAD, and SARC. DNASE1L3 was significantly associated with CCR7/CCL19 in cancers. DNASE1L3 was downregulated in HCC and STAD tissues as demonstrated by qRT-PCR, as well as in HCC and LUAD samples, as shown by immunohistochemistry.

Conclusion: DNASE1L3 has potential to serve as a prognostic biomarker in cancer of the breast, kidney, liver, stomach, lung adenocarcinoma and sarcoma. Down-regulation of DNASE1L3 may participate in immune escape via CCR7/CCL19 axis.

Keywords: DNASE1L3, CCR7, CCL19, prognosis, immune infiltration, tumor

\section{Introduction}

Immunotherapy has shed light on treatment of cancer. Accumulating evidence has indicated that the immune system plays a key role in human cancer, ${ }^{1,2}$ so immunotherapy is one of the most promising directions in cancer treatment. ${ }^{3,4}$ Recently, immune checkpoint blockade has exhibited significant antitumor effects in several malignancies, such as melanoma, renal cell cancer, hepatocellular carcinoma (HCC), biliary tract carcinoma, gastric cancer, and head and neck cancer. ${ }^{5-7}$ Despite unprecedented efficacy of immune checkpoint blockade, majority of patients showed no response to immunotherapy, while some patients who had encouraging initial response, acquired resistance over time. Thus, a better understanding of underlying mechanisms is urgent for immunotherapy for cancer. 
For example, infiltrating lymphocytes in tumors may affect prognosis and response to immunotherapy. ${ }^{8-10}$ Collectively, elucidating various factors modulating immune infiltration and identifying new immunotherapeutic targets are essential to improve efficacy of cancer immunotherapy.

Deoxyribonuclease 1 like 3 (DNASE1L3) is a member of the deoxyribonuclease 1 family, with similar constituent to DNase, ${ }^{11,12}$ which was first identified from thymocytes of rat in $1994^{13}$ and cloned as homolog of human DNASE1L3 in 1998. ${ }^{14}$ DNASE1L3 is important for DNA catabolism and apoptosis. $^{15,16}$ Previous studies indicated that DNASE1L3 participated in signal transduction in breast cancer, including intracellular signaling cascade, receptor activity, and GTPase regulator. ${ }^{17}$ Moreover, DNASE1L3 is a prognostic indicator of HCC. ${ }^{18}$ Above all, DNASE1L3 probably plays a role in tumorigenesis.

In addition, DNASE1L3 secreted by dendritic cells (DCs), macrophages, and neutrophils ${ }^{16,19}$ can be detected in circulation. DNASE1L3 is involved in programmed cell death during development, ${ }^{20}$ immune response related to neutrophil activation, acute inflammatory response, and neutrophil mediated cytotoxicity. ${ }^{21,22}$ These findings indicate functions of DNASE1L3 in the immune system.

Considering interactions among cancer, immune system and DNASE1L3, we measured its expression, estimated its prognostic value, and explored its association with immune infiltration in human cancers comprehensively.

\section{Materials and Methods}

\section{Genetic Analysis Using GEPIA2}

Using GEPIA2 (http://gepia2.cancer-pku.cn/\#index) ${ }^{23}$ the most differential survival and expression genes were explored in 33 different human cancers in TCGA and GTEx. DNASE1L3 was identified to be downregulated in almost all human cancers.

\section{Genetic Analysis Using Oncomine}

Using Oncomine gene expression array database (https:// www.oncomine.org/resource/login.html), ${ }^{24}$ mRNA level of DNASE1L3 gene was assessed in human cancers. The threshold was defined as: $P=0.001$, fold change $=2.0$, and gene ranking of all.

\section{Prognostic Analysis Using PrognoScans}

Using PrognoScan (http://dna00.bio.kyutech.ac.jp/ PrognoScan/), ${ }^{25}$ prognostic value of DNASE1L3 was evaluated in human cancers. The threshold was defined as Cox
$P<0.05$, describing hazard ratio (HR) with $95 \%$ confidence intervals $(\mathrm{CI})$.

\section{Prognostic Analysis Using Kaplan-Meier Plotter}

Using Kaplan-Meier plotter (http://www.kmplot.com/ana lysis/index.php? $\mathrm{p}=$ background $),{ }^{26,27}$ prognostic value of DNASE1L3 was estimated in human cancers. The HR with $95 \%$ CI was marked.

\section{bc-GenEcMiner V4.5 Database Analysis}

Using Breast Cancer Gene-Expression Miner v4.5 (http:// bcgenex.centregauducheau.fr/BC-GEM/GEM-Accueil. php?js $=1),{ }^{28,29}$ the prognostic role of DNASE1L3 was explored in breast cancer. bc-GenExMiner v4.5 is a statistical mining tool of published annotated breast cancer transcriptomic data (DNA microarrays $[\mathrm{n}=$ 10,716] and RNA-seq [ $\mathrm{n}=4712]$ ), which estimates prognostic values of specific genes in breast cancer.

\section{TIMER Database Analysis}

Using Tumor Immune Estimation Resource (TIMER) (https://cistrome.shinyapps.io/timer/), ${ }^{30,31}$ differential gene expression between multiple cancers and related normal tissues, as well as association between two molecules in cancers could be analyzed. The Cancer Genome Atlas (TCGA) was used to estimate immune infiltration. Moreover, gene expression against tumor purity can be normalized through TIMER. $^{32}$ We analyzed DNASE1L3 expression across 32 types of human cancers as well as the relationship between DNASE1L3 and immune infiltration. Association between DNASE1L3 and multiple genetic markers of tumorinfiltrating immune cells was analyzed. ${ }^{33-36}$ Then, Spearman correlation and estimated statistical significance of a pair of defined genes in one cancer type were generated through correlation module. DNASE1L3 was applied to $\mathrm{x}$-axis, and related markers were represented on $\mathrm{y}$-axis. Gene expression level was displayed with $\log 2$ RSEM.

\section{DNASEIL3 Expression in HCC, Stomach and Lung Adenocarcinoma}

This study was approved by Ethical Committee of Beijing Shijitan Hospital and performed by complying with all relevant ethical regulations [No. sjtkyll-lx-2019(36)]. Written informed consent from each patient was obtained prior to study commencement, and all processes were conducted in accordance with the Declaration of Helsinki. 
qRT-PCR was conducted to measure expression of DNASE1L3 in HCC samples and adjacent normal tissues $(n=22)$, as well as the expression of DNASE1L3, CCR7, and CCL19 in STAD samples and adjacent normal tissues $(\mathrm{n}=17)$, which were collected in Beijing Shijitan Hospital. Briefly, total RNA was isolated using E.Z.N.A Total RNA Kit II (OMEGA R6934-02) according to the manufacturer's protocol. ReverTra Ace qPCR RT Kit (TOYOBO FSQ-101) was used to synthesize the first-strand cDNA. Real-time qPCR was performed on Quant Studio 6 Real Time PCR System with SYBR (Thermo Fisher Scientific). $18 \mathrm{~S}$ was used as reference gene. The sequences of primers were as follows: DNASE1L3 Forward: AGCCC TTTGTGGTCTGGTTC, Reverse: TCCTTAACGGATG TCTCTGGG; CCR7 Forward: TGAGGTCACGGACGAT TACAT, Reverse: GTAGGCCCACGAAACAAATGAT. CCL19 Forward: CTGCTGGTTCTCTGGACTTCC, Reverse: AGGGATGGGTTTCTGGGTCA. 18S Forward: GTAACCCGTTGAACCCCATT, Reverse: CCATCCAA TCGGTAGTAGCG.

Immunohistochemistry was used to explore protein expression of DNASE1L3 in formalin-fixed paraffinembedded (FFPE) tissues collected at Beijing Shijitan Hospital. Briefly, 12 paired HCC and adjacent normal tissue FFPE samples, 20 paired lung adenocarcinoma and adjacent normal tissue FFPE samples were collected from the Department of Pathology. The primary and secondary antibodies were purchased from Abcam (ab203669, ab205718). Immunohistochemistry procedures followed the manufacturer's instructions (https://www.abcam.com/ protocols/immunostaining-paraffin-frozen-free-floatingprotocol). The expression level of DNASE1L3 in each sample (collected from 3 random fields, 200×) was evaluated by AOD with Image J software. The clinical information was listed in Supplementary Tables 4 and 5.

\section{Statistical Analysis}

Data were analyzed by GraphPad Prism software (version 7.04; GraphPad Prism software Inc., San Diego, CA). Survival curves were generated by PrognoScan, KaplanMeier plot, GEPIA2 and bc-GenEcMiner v4.5. Data from Oncomine were represented as P-values, $t$-test values, fold changes, ranks and sample numbers. Results from KaplanMeier plots, PrognoScan, GEPIA2 and bc-GenEcMiner v4.5 were represented as $\mathrm{HR}, 95 \% \mathrm{CI}$ and $P$ or Cox $P$-values from Log rank tests. Association between two genes was evaluated by Spearman correlation and absolute value of correlation was defined as follows: $0.00-0.19$ : very weak; $0.20-0.39$ : weak; $0.40-0.59$ : moderate; $0.60-0.79$ : strong; $0.80-1.0$ : very strong. Paired $t$-test was used to compare DNASE1L3 expression levels between tumor and adjacent normal samples. A $P<0.05$ was considered as statistically significant.

\section{Results}

\section{The mRNA Expression Profiling of DNASEIL3 in Various Cancers}

The DNASE1L3 mRNA levels in human cancers and adjacent normal tissues were analyzed by Oncomine database. DNASE1L3 was downregulated in breast, cervical, colorectal, esophageal, gastric, head and neck, kidney, liver, lung, ovarian, pancreatic cancer, mesothelioma, leukemia, sarcoma, pleural malignant mesothelioma and pleural malignant fibrous histiocytoma. On the other hand, only lymphoma had higher expression of DNASE1L3 (Figure 1A). More information on DASE1L3 expression was collected in Supplementary Table 1.

Moreover, expression level of DNASE1L3 was also analyzed in TCGA database through TIMER. DNASE1L3 was downregulated in bladder urothelial carcinoma (BLCA), breast invasive carcinoma (BRCA), cholangiocarcinoma (CHOL), head and neck squamous cell carcinoma (HNSC), kidney chromophobe (KICH), kidney renal clear cell carcinoma (KIRC), kidney renal papillary cell carcinoma (KIRP), liver hepatocellular carcinoma (LIHC), lung adenocarcinoma (LUAD), lung squamous cell carcinoma (LUSC), prostate adenocarcinoma (PRAD), rectum adenocarcinoma (READ), stomach adenocarcinoma (STAD), thyroid carcinoma (THCA), and uterine corpus endometrial carcinoma (UCEC) compared with adjacent normal tissues (Figure 1B).

\section{Prognostic Value of DNASEIL3 in Human Cancers}

Based on PrognoScan, DNASE1L3 might impact prognosis in blood cancer (follicular lymphoma and multiple myeloma), breast cancer, colorectal cancer, lung adenocarcinoma, head and neck cancer (squamous cell carcinoma), ovarian cancer, and skin cancer (melanoma). Lower expression of DNASE1L3 was related to worse prognosis in these cancers (Figure 2A-H, Supplementary Figure 1). In GSE31210 cohort, 204 lung adenocarcinoma patients were divided into DANSE1L3 higher expression group $(n=108)$ and lower expression group $(n=196)$, a lower level of DNASE1L3 was correlated with poorer overall survival (OS, $\mathrm{HR}=0.61, P=0.0212$ ), $\mathrm{HR}=0.61$ means the 


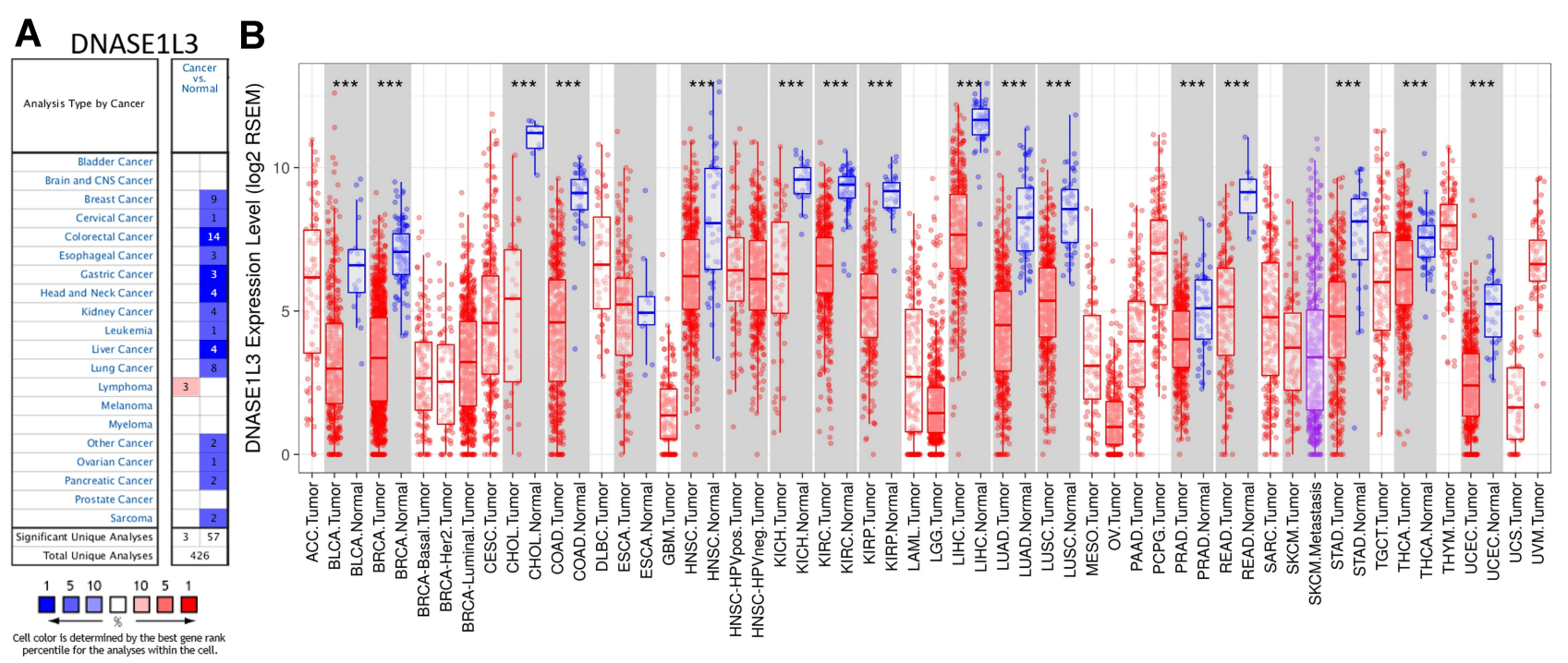

Figure I The expression of DNASEIL3 in different human cancers. (A) The expression of DNASEIL3 between cancer and normal tissue in the OncoMine database. (B) DNASEIL3 expression levels in multiple human cancers from TCGA database were analyzed by TIMER. ***P $<0.001$.

Abbreviations: ACC, adrenocortical carcinoma; BLCA, bladder urothelial carcinoma; BRCA, breast invasive carcinoma; CESC, cervical squamous cell carcinoma and endocervical adenocarcinoma; CHOL, cholangio carcinoma; COAD, colon adenocarcinoma; DLBC, lymphoid neoplasm diffuse large B-cell lymphoma; ESCA, esophageal carcinoma; GBM, glioblastoma multiforme; HNSC, head and neck squamous cell carcinoma; KICH, kidney chromophobe; KIRC, kidney renal clear cell carcinoma; KIRP, kidney renal papillary cell carcinoma; LAML, acute myeloid leukemia; GG, brain lower grade glioma; LIHC, liver hepatocellular carcinoma; LUAD, lung adenocarcinoma; LUSC, lung squamous cell carcinoma; MESO, mesothelioma; OV, ovarian serous cystadenocarcinoma; PAAD, pancreatic adenocarcinoma; PCPG, pheochromocytoma and paraganglioma; PRAD, prostate adenocarcinoma; READ, rectum adenocarcinoma; SARC, sarcoma; SKCM, skin cutaneous melanoma; STAD, stomach adenocarcinoma; TGCT, testicular germ cell tumors; THCA, thyroid carcinoma; THYM, thymoma; UCEC, uterine corpus endometrial carcinoma; UCS, uterine carcinosarcoma; UVM, uveal melanoma.

probability of OS in lower group/higher group $=0.61$ (Figure 2E).

Based on Kaplan-Meier plotter, DNASE1L3 might influence prognosis in bladder cancer, breast cancer, gastric carcinoma, liver cancer, lung adenocarcinoma, lung squamous cell carcinoma, ovarian cancer, cervical squamous cell carcinoma, head-neck squamous cell carcinoma, pancreatic adenocarcinoma, kidney renal clear cell carcinoma, sarcoma and mesothelioma. In all of these cancers/ tumors except bladder cancer, lower expression of DNASE1L3 indicated worse prognosis (Figure 2I-P, Supplementary Figure 2).

In addition, prognostic value of DNASE1L3 was also estimated through GEPIA (Supplementary Figure 3). Interestingly, lower expression of DNASE1L3 in ACC, KICH, KIRC, LIHC, LUAD, PAAD, PCPG, and SARC correlated with poorer prognosis (reduced OS). By contrast, higher expression of DNASE1L3 in BCLA and UVM correlated with worse prognosis. As for relapse free survival (RFS), lower expression of DNASE1L3 in CESC, CHOL, KICH, KIRC, KIRP, LIHC, PAAD, and THCA correlated with poorer prognosis, whereas higher expression of DNASE1L3 in UVM correlated with worse prognosis (Supplementary Figure 3). Lastly, using bc-
GenEcMiner v4.5 Database, prognostic value of DNASE1L3 was indicated in breast cancer. Lower expression of DNASE1L3 correlated with poorer OS and disease free survival (DFS) in breast cancer (Figure 3). Collectively, all these results indicated that DNASE1L3 might harbor significant prognostic value in various cancers.

\section{DNASEIL3 Correlates with Immune Infiltration in Various Cancers}

Tumor-infiltrating lymphocytes affect prognosis and response to immunotherapy of cancers. ${ }^{8-10}$ A previous study indicated that circulating DNASE1L3 could be secreted by dendritic cells (DC), macrophages, and neutrophils. Thus, DNASE1L3 may be connected with immune infiltration in various cancers. Then, TIMER was used to analyze relationship of DNASE1L3 expression with immune infiltration in multiple cancers. Interestingly, DNASE1L3 correlated with immune infiltration in diverse cancers. DNASE1L3 expression generally correlated with tumor purity in 31 human cancers, with B cells in 24 human cancers, with CD8+T cells in 19 human cancers, with macrophages in 13 human cancers, with neutrophils in 17 human cancers, as well as with 

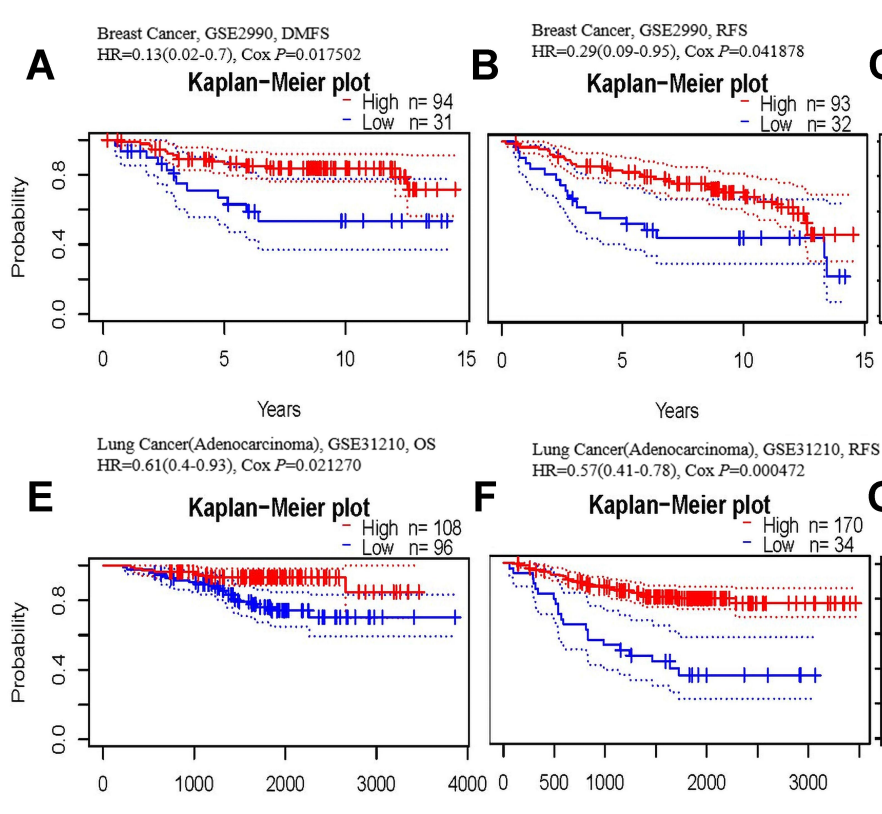

Years

Lung Cancer(Adenocarcinoma), GSE31210, RFS $\mathrm{HR}=0.57(0.41-0.78), \mathrm{Cox} P=0.000472$

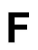

Kaplan-Meier plot

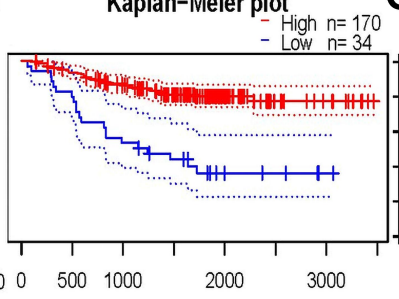

Breast Cancer, GSE1456-GPL96, RFS

Kaplan-Meier plot

Breast Cancer, GSE6532-GPL570, RFS

D $H R=0.36(0.14-0.88), \operatorname{Cox} P=0.025044$

Kaplan-Meier plot
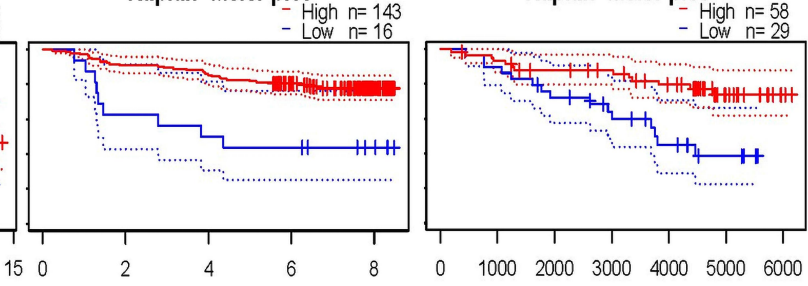

Years

Days

Days

Days

I

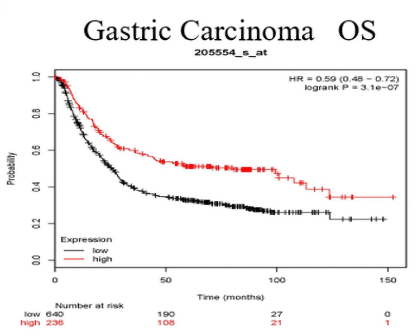

J

Gastric Carcinoma PPS

Ovarian Cancer, GSE9891, OS $\mathrm{HR}=0.36(0.14-0.95)$, $\operatorname{Cox} P=0.038067$

19234, OS

G Kaplan-Meier plot ${ }_{-}$High $n=97$

$\mathrm{HR}=0.45(0.24-0.84), \operatorname{Cox} P=0.011803$

H Kaplan-Meier plot

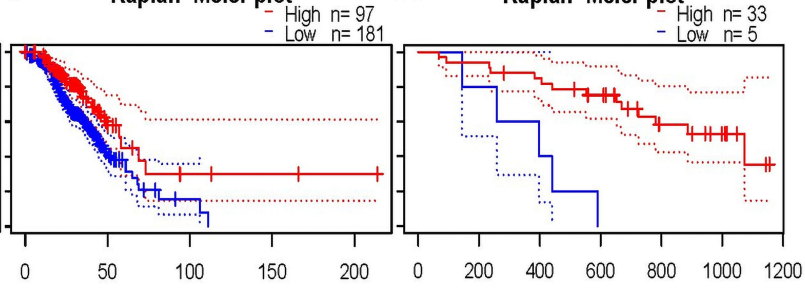
- Low $n=5$

M

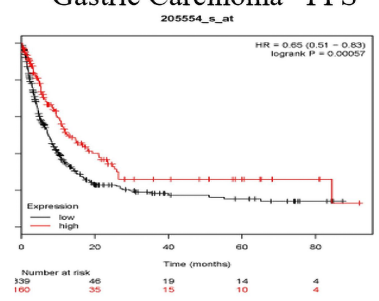

K Kidney renal clear cell $\underset{\text { DAASEIL3 }}{\operatorname{arcinoma}}$ OS

L Kidney renal

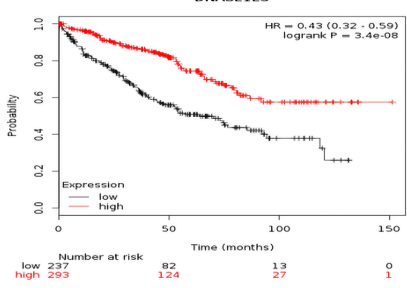
clear cell ç carcinoma RFS

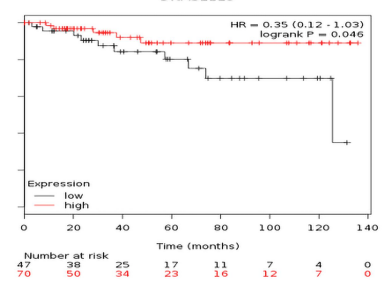

$\mathbf{N}$

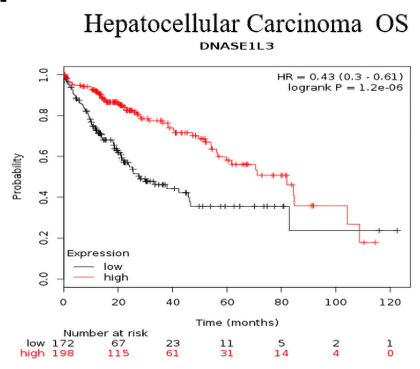

Hepatocellular Carcinoma RFS

0

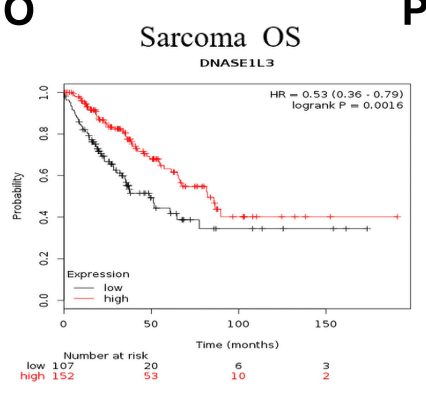

$\mathbf{P}$

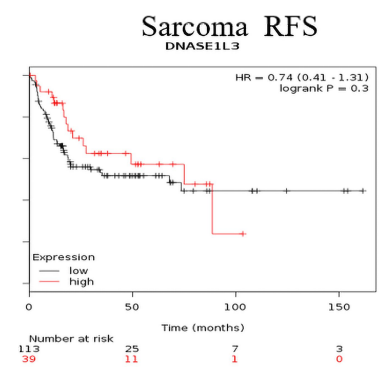

Figure 2 The prognostic value of DNASEIL3 in various human cancers. Kaplan-Meier survival curves were used to analyze the prognostic value of DNASEIL3 in various human cancers. (A-H) Came from PrognoScan database, (I-P) came from Kaplan-Meier plotter database. (A-D) Survival curves of DMFS and RFS in three breast cancer cohorts (GSE2990, GSEI456-GPL96, GSE6532-GPL570). (E, F) Survival curves of OS and RFS in one lung adenocarcinoma cohort (GSE3 I2I0). (G H) Survival curves of OS in one ovarian cancer cohort (GSE989I) and one skin cancer (GSEI9234). (I-P) Survival curves of OS and PPS/RFS in gastric carcinoma, kidney renal clear cell carcinoma, hepatocellular carcinoma, and sarcoma respectively.

Abbreviations: OS, overall survival; RFS, relapse-free survival; PPS, post-progression survival; DMFS, disease metastasis-free survival.

dendritic cells in human cancers (Figure 4, Supplementary Figure 4). For 31 types of cancers with significant association between DNASE1L3 expression and tumor purity, relationship between immune infiltration and DNASE1L3 was defined when 4 or more types of immune cells correlated with DNASE1L3. In this way, 14 types of cancers were identified. Moreover, DNASE1L3 harbored prognostic value in 9 out of 14 cancers, including BRCA, HNSC,
KIRP, LIHC, LUAD, SARC, SKCM, STAD, and UCEC (Figure 4). Taking BRCA as an example, DNASE1L3 expression correlated with tumor purity negatively, whereas with infiltration of CD8 $+\mathrm{T}$ cells, CD4+T cells, neutrophils and DCs (but not B cell and macrophage) positively. Similar results were observed in the other 8 types of cancers, except LIHC. Therefore, DNASE1L3 might participate in immune infiltration in these 9 types 

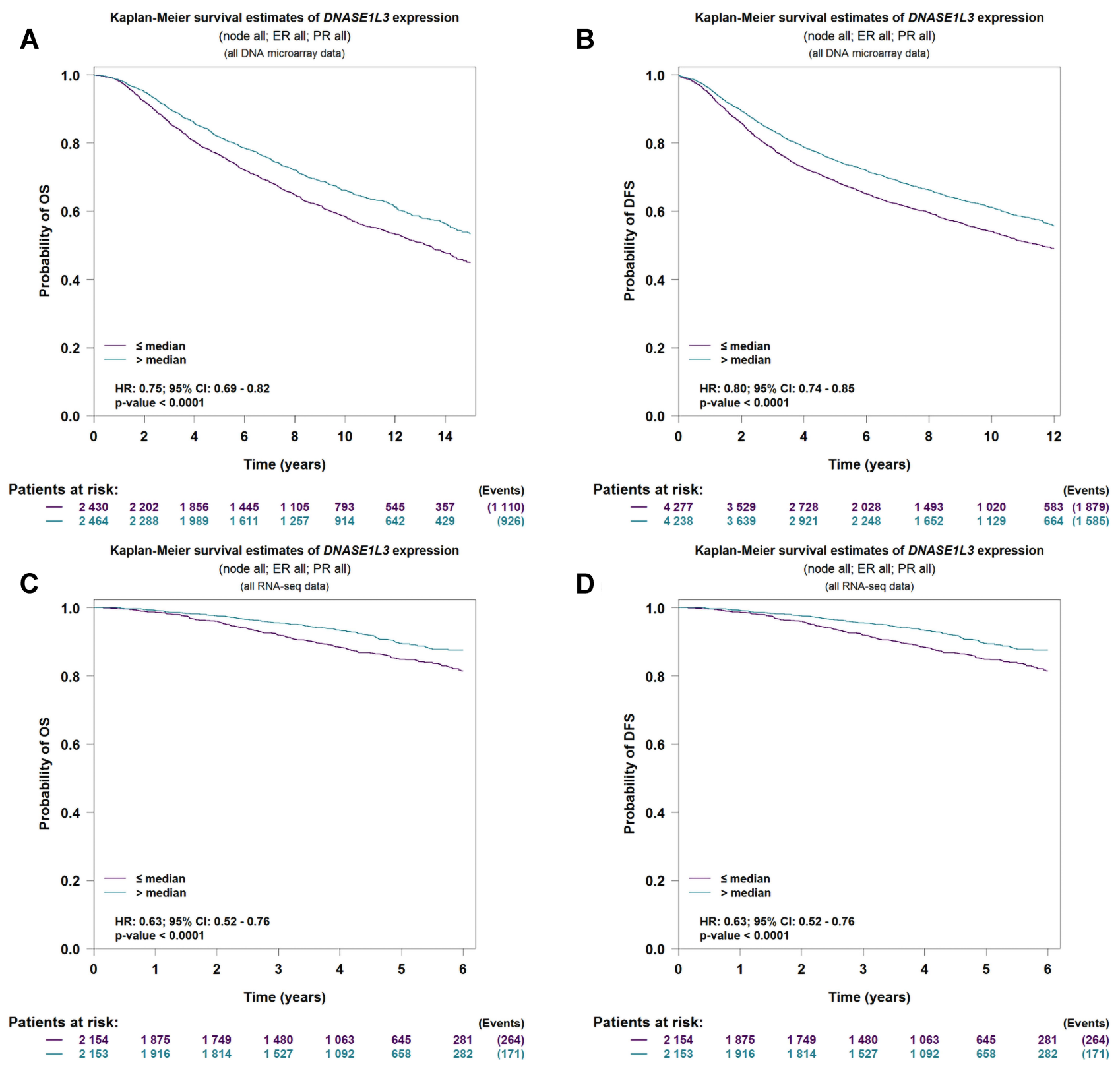

Figure 3 The prognostic value of DNASEIL3 in breast cancer. The prognostic value and the expression of DNASEIL3 in breast cancer were evaluated through bcGenExMiner v4.5. (A B) Came from DNA microarray data collected in bc-GenExMiner v4.5, (C D) Came from RNA microarray data collected in bc-GenExMiner v4.5. (A and C) The lower expression of DNASEIL3 had poor probability of OS in breast cancer patients; (B and D) The lower expression of DNASEIL3 had poor probability of DFS in breast cancer patients.

Abbreviations: OS, overall survival; DFS, disease-free survival.

of cancers, especially for $\mathrm{T}$ cells (both $\mathrm{CD} 4+$ and $\mathrm{CD} 8+$ ) and DCs.

\section{DNASEIL3 Correlates with Immune Markers in Various Cancers}

To decipher the interaction of DNASE1L3 with immune infiltrating cells, association between DNASE1L3 expression and markers of various immune cells in BRCA, HNSC, KIRP, LIHC, LUAD, SARC, SKCM, STAD, and
UCEC was evaluated through TIMER and GEPIA. As previously described, ${ }^{33}$ immune markers of CD8+ T cells, T cells (general), Th1 cells, Th2 cells, Tfh cells, Th17 cells, and regulatory $\mathrm{T}$ cells (Tregs), exhausted $\mathrm{T}$ cells, B cells, monocytes, tumor-associated macrophages (TAMs), M1 and M2 macrophages, neutrophils, NK cells and DCs were selected to analyze individual association with DNASE1L3 in the previously mentioned 9 cancers (Table 1, Supplementary Table 2). Interestingly, 


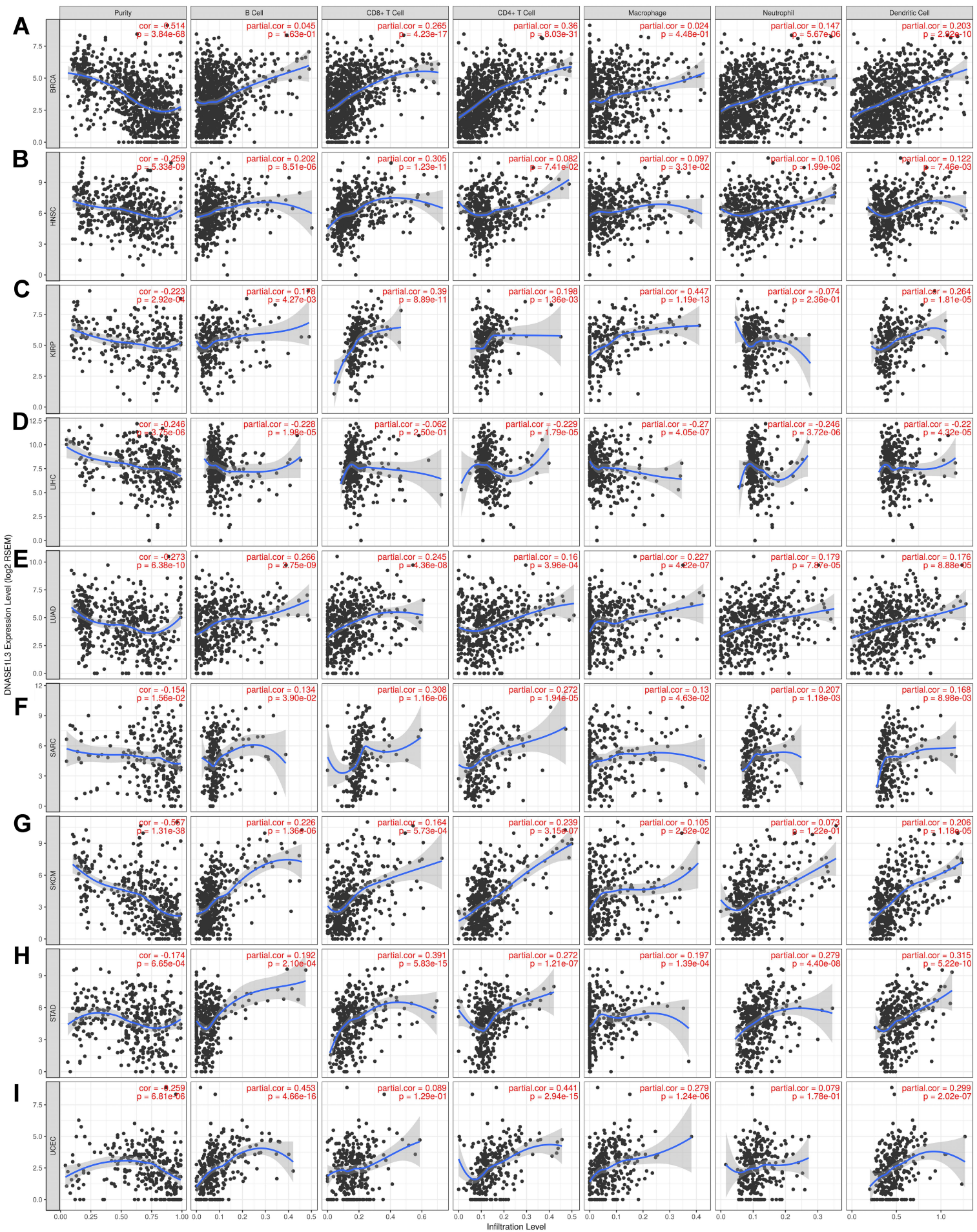

Figure 4 The relationship between DNASEIL3 expression and immune infiltration level. The relationship between DNASEIL3 expression and immune infiltration level in (A) breast invasive carcinoma (BRCA), (B) head and neck squamous cell carcinoma (HNSC), (C) kidney renal clear cell carcinoma (KIRP), (D) liver hepatocellular carcinoma (LIHC), (E) lung adenocarcinoma (LUAD), (F) sarcoma (SARC), (G) skin cutaneous melanoma (SKCM), (H) stomach adenocarcinoma (STAD), (I) uterine carcinoma (UCEC). The expression of DNASEIL3 level was significantly negatively correlated with tumor purity with these 9 cancers. In BRCA, the expression of DNASEIL3 showed positive correlation with CD8+T cell, CD4+T cell, neutrophil and dendritic cell, except B cell and macrophage (A). In HNSC, DNASEI L3 was positively correlated with B cell, CD8+T cell, CD4+T cell, macrophage, neutrophil and dendritic cell, except CD4+ T cell (B). In KIRP, DNASEIL3 showed positive correlation with B cell, CD8+T cell, CD4+T cell, macrophage, and dendritic cell, other than neutrophil (C). In LIHC, DNASEIL3 showed negative correlation with B cell, CD4+T cell, macrophage, neutrophil and dendritic cell, other than CD8+T cell (D). In LUAD, DNASEI L3 showed positive correlation with B cell, CD8+T cell, CD4+T cell, macrophage, neutrophil and dendritic cell (E). In SARC, DNASEI L3 showed positive correlation with B cell, CD8+T cell, CD4+T cell, macrophage, neutrophil and dendritic cell (F). In SKCM, DNASEIL3 showed positive correlation with B cell, CD8+T cell, CD4+T cell, macrophage, neutrophil and dendritic cell (G). In STAD, DNASEIL3 showed positive correlation with B cell, CD8+T cell, CD4+T cell, macrophage and dendritic cell, other than neutrophil (H). In UCEC, DNASEIL3 showed positive correlation with B cell, CD8+T cell, CD4+T cell, macrophage, neutrophil and dendritic cell (I). 
Table I Correlation Analysis Between DNASEIL3 and Markers of Immune Cells Through TIMER

\begin{tabular}{|c|c|c|c|c|c|c|c|c|c|c|c|c|c|}
\hline \multirow[t]{3}{*}{ Description } & \multirow[t]{3}{*}{ Gene Markers } & \multicolumn{4}{|c|}{$\operatorname{BRCA}(n=1093)$} & \multicolumn{4}{|c|}{ STAD $(n=4 \mid 5)$} & \multicolumn{4}{|c|}{$\operatorname{LUAD}(n=5 \mid 5)$} \\
\hline & & \multicolumn{2}{|c|}{ None } & \multicolumn{2}{|c|}{ Purity } & \multicolumn{2}{|c|}{ None } & \multicolumn{2}{|c|}{ Purity } & \multicolumn{2}{|c|}{ None } & \multicolumn{2}{|c|}{ Purity } \\
\hline & & Cor & $P$ & Cor & $\mathbf{P}$ & Cor & $P$ & Cor & $\mathbf{P}$ & Cor & $P$ & Cor & $P$ \\
\hline \multirow[t]{2}{*}{$\mathrm{CD} 8+\mathrm{T}$ cell } & CD8A & 0.555 & $* * * *$ & 0.389 & $* * * *$ & 0.387 & $* * * *$ & 0.358 & $* * * *$ & 0.328 & $* * * *$ & 0.251 & $* * * *$ \\
\hline & CD8B & 0.515 & $* * * *$ & 0.354 & $* * * *$ & 0.339 & $* * * *$ & 0.308 & $* * * *$ & 0.297 & $* * * *$ & 0.225 & $* * * *$ \\
\hline \multirow[t]{3}{*}{ T cell (genenral) } & CD3D & 0.567 & $* * * *$ & 0.389 & $* * * *$ & 0.493 & $* * * *$ & 0.464 & $* * * *$ & 0.337 & $* * * *$ & 0.243 & $* * * *$ \\
\hline & CD3E & 0.590 & $* * * *$ & 0.415 & $* * * *$ & 0.475 & $* * * *$ & 0.444 & $* * * *$ & 0.397 & $* * * *$ & 0.320 & $* * * *$ \\
\hline & CD2 & 0.530 & $* * * *$ & 0.346 & $* * * *$ & 0.458 & $* * * *$ & 0.430 & $* * * *$ & 0.379 & $* * * *$ & 0.299 & $* * * *$ \\
\hline \multirow[t]{2}{*}{$B$ cell } & CDI9 & 0.495 & $* * * *$ & 0.317 & $* * * *$ & 0.478 & $* * * *$ & 0.440 & $* * * *$ & 0.399 & $* * * *$ & 0.320 & $* * * *$ \\
\hline & CD79A & 0.524 & $* * * *$ & 0.337 & $* * * *$ & 0.494 & $* * * *$ & 0.468 & $* * * *$ & 0.290 & $* * * *$ & 0.201 & $* * * *$ \\
\hline \multirow[t]{2}{*}{ Monocyte } & CD86 & 0.269 & $* * * *$ & 0.070 & $*$ & 0.292 & $* * * *$ & 0.256 & $* * * *$ & 0.286 & $* * * *$ & 0.199 & $* * * *$ \\
\hline & CDII5(CSFIR) & 0.409 & $* * * *$ & 0.216 & $* * * *$ & 0.356 & $* * * *$ & 0.327 & $* * * *$ & 0.253 & $* * * *$ & 0.170 & $* * *$ \\
\hline \multirow[t]{3}{*}{ TAM } & $\mathrm{CCL} 2$ & 0.347 & $* * * *$ & 0.179 & $* * * *$ & 0.162 & $* * *$ & 0.119 & $*$ & 0.144 & $* *$ & 0.061 & 0.177 \\
\hline & CD68 & 0.212 & $* * * *$ & 0.016 & 0.607 & 0.219 & $* * * *$ & 0.197 & $* * *$ & 0.310 & $* * * *$ & 0.247 & $* * * *$ \\
\hline & ILIO & 0.274 & $* * * *$ & 0.091 & $* *$ & 0.194 & $* * * *$ & 0.135 & $* *$ & 0.390 & $* * * *$ & 0.316 & $* * * *$ \\
\hline \multirow[t]{3}{*}{ MI Macrophage } & INOS(NOS2) & 0.066 & $*$ & 0.037 & 0.250 & 0.106 & $*$ & 0.098 & 0.056 & 0.158 & $* * *$ & 0.103 & $*$ \\
\hline & IRF5 & 0.213 & $* * * *$ & 0.095 & $* *$ & 0.123 & $*$ & 0.070 & 0.174 & 0.142 & $* *$ & 0.046 & 0.310 \\
\hline & COX2(PTGS2) & 0.404 & $* * * *$ & 0.274 & $* * * *$ & 0.056 & 0.259 & 0.094 & 0.066 & 0.077 & 0.079 & 0.077 & 0.086 \\
\hline \multirow[t]{3}{*}{ M2 Macrophage } & CDI63 & 0.231 & $* * * *$ & 0.051 & 0.110 & 0.190 & $* * * *$ & 0.168 & $* *$ & 0.257 & $* * * *$ & 0.175 & $* * * *$ \\
\hline & VSIG4 & 0.253 & $* * * *$ & 0.086 & $* *$ & 0.114 & $*$ & 0.094 & 0.066 & 0.266 & $* * * *$ & 0.198 & $* * * *$ \\
\hline & MS4A4A & 0.394 & $* * * *$ & 0.211 & $* * * *$ & 0.290 & $* * * *$ & 0.263 & $* * * *$ & 0.359 & $* * * *$ & 0.291 & $* * * *$ \\
\hline \multirow[t]{3}{*}{ Neutrophils } & CD66b(CEACAM8) & 0.063 & $*$ & 0.096 & $* *$ & 0.129 & $* *$ & 0.136 & $* *$ & 0.293 & $* * * *$ & 0.291 & $* * * *$ \\
\hline & CDIIb(ITGAM) & 0.211 & $* * * *$ & 0.025 & 0.424 & 0.235 & $* * * *$ & 0.202 & $* * * *$ & 0.188 & $* * * *$ & 0.106 & $*$ \\
\hline & CCR7 & 0.621 & $* * * *$ & 0.464 & $* * * *$ & 0.567 & $* * * *$ & 0.532 & $* * * *$ & 0.515 & $* * * *$ & 0.453 & $* * * *$ \\
\hline \multirow[t]{7}{*}{ Natural killer cell } & KIR2DLI & 0.200 & $* * * *$ & 0.090 & $* *$ & 0.174 & $* * *$ & 0.160 & $* *$ & 0.151 & $* * *$ & 0.116 & $* *$ \\
\hline & KIR2DL3 & 0.224 & $* * * *$ & 0.098 & $* *$ & 0.143 & $* *$ & 0.108 & $*$ & 0.161 & $* * *$ & 0.097 & $*$ \\
\hline & KIR2DL4 & 0.230 & $* * * *$ & 0.087 & $* *$ & 0.178 & $* * *$ & 0.160 & $* *$ & 0.017 & 0.695 & 0.042 & 0.357 \\
\hline & KIR3DLI & 0.299 & $* * * *$ & 0.164 & $* * * *$ & 0.221 & $* * * *$ & 0.236 & $* * * *$ & 0.162 & $* * *$ & 0.114 & $*$ \\
\hline & KIR3DL2 & 0.323 & $* * * *$ & 0.175 & $* * * *$ & 0.199 & $* * * *$ & 0.185 & $* * *$ & 0.151 & $* * *$ & 0.092 & $*$ \\
\hline & KIR3DL3 & 0.158 & $* * * *$ & 0.083 & $* *$ & 0.132 & $* *$ & 0.143 & $* *$ & 0.040 & 0.366 & 0.054 & 0.230 \\
\hline & KIR2DS4 & 0.234 & $* * * *$ & 0.113 & $* * *$ & 0.170 & $* * *$ & 0.170 & $* * *$ & 0.106 & $*$ & 0.054 & 0.231 \\
\hline Dendritic cell & HLA-DPBI & 0.536 & $* * * *$ & 0.353 & $* * * *$ & 0.358 & $* * * *$ & 0.321 & $* * * *$ & 0.393 & $* * * *$ & 0.334 & $* * * *$ \\
\hline & HLA-DQBI & $0.34 I$ & $* * * *$ & 0.172 & $* * * *$ & 0.276 & $* * * *$ & 0.225 & $* * * *$ & 0.239 & $* * * *$ & 0.162 & $* * *$ \\
\hline & HLA-DRA & $0.44 I$ & $* * * *$ & $0.24 I$ & $* * * *$ & 0.338 & $* * * *$ & 0.308 & $* * * *$ & 0.349 & $* * * *$ & 0.283 & $* * * *$ \\
\hline & HLA-DPAI & 0.475 & $* * * *$ & 0.293 & $* * * *$ & 0.337 & $* * * *$ & 0.303 & $* * * *$ & 0.346 & $* * * *$ & 0.286 & $* * * *$ \\
\hline & BDCA-I (CDIC) & 0.713 & $* * * *$ & 0.598 & $* * * *$ & $0.57 \mid$ & $* * * *$ & 0.532 & $* * * *$ & 0.385 & $* * * *$ & 0.343 & $* * * *$ \\
\hline & BDCA4(NRPI) & 0.309 & $* * * *$ & 0.153 & $* * * *$ & 0.177 & $* * *$ & 0.134 & $* *$ & 0.008 & 0.863 & 0.035 & 0.432 \\
\hline & CDIIc(ITGAX) & 0.304 & $* * * *$ & 0.102 & $* *$ & 0.260 & $* * * *$ & 0.218 & $* * * *$ & 0.270 & $* * * *$ & 0.184 & $* * * *$ \\
\hline Thl & T-bet(TBX2I) & 0.558 & $* * * *$ & 0.388 & $* * * *$ & 0.417 & $* * * *$ & 0.389 & $* * * *$ & 0.349 & $* * * *$ & 0.264 & $* * * *$ \\
\hline & STATI & 0.054 & 0.075 & 0.071 & $*$ & 0.084 & 0.087 & 0.083 & 0.105 & 0.095 & $*$ & 0.013 & 0.777 \\
\hline & STAT4 & 0.542 & $* * * *$ & 0.350 & $* * * *$ & 0.466 & $* * * *$ & $0.44 I$ & $* * * *$ & 0.281 & $* * * *$ & 0.196 & $* * * *$ \\
\hline & IFN- $\gamma($ IFNG $)$ & 0.309 & $* * * *$ & 0.141 & $* * * *$ & 0.156 & $* *$ & 0.137 & $* *$ & 0.128 & $* *$ & 0.042 & 0.349 \\
\hline & TNF- $\alpha$ (TNF) & 0.084 & $* *$ & 0.017 & 0.603 & 0.174 & $* * *$ & 0.130 & $*$ & 0.171 & $* * * *$ & 0.070 & 0.122 \\
\hline Th2 & GATA3 & 0.102 & $* * *$ & 0.058 & 0.070 & 0.269 & $* * * *$ & 0.242 & $* * * *$ & 0.162 & $* * *$ & 0.077 & 0.088 \\
\hline & STAT5A & 0.423 & $* * * *$ & 0.291 & $* * * *$ & 0.262 & $* * * *$ & 0.228 & $* * * *$ & 0.316 & $* * * *$ & 0.232 & $* * * *$ \\
\hline
\end{tabular}

(Continued) 
Table I (Continued).

\begin{tabular}{|c|c|c|c|c|c|c|c|c|c|c|c|c|c|}
\hline \multirow[t]{4}{*}{ Description } & \multirow{4}{*}{$\begin{array}{c}\text { Gene Markers } \\
\\
\text { STAT6 } \\
\text { ILI3 }\end{array}$} & \multicolumn{4}{|c|}{ BRCA $(n=1093)$} & \multicolumn{4}{|c|}{ STAD $(n=4 \mid 5)$} & \multicolumn{4}{|c|}{$\operatorname{LUAD}(n=5 \mid 5)$} \\
\hline & & \multicolumn{2}{|c|}{ None } & \multicolumn{2}{|c|}{ Purity } & \multicolumn{2}{|c|}{ None } & \multicolumn{2}{|c|}{ Purity } & \multicolumn{2}{|c|}{ None } & \multicolumn{2}{|c|}{ Purity } \\
\hline & & 0.303 & $* * * *$ & 0.276 & $* * * *$ & 0.179 & $* * *$ & 0.164 & $* *$ & 0.165 & $* * *$ & 0.183 & $* * * *$ \\
\hline & & 0.169 & $* * * *$ & 0.094 & $* *$ & 0.019 & 0.705 & -0.018 & 0.722 & 0.198 & $* * * *$ & 0.159 & $* * *$ \\
\hline \multirow[t]{2}{*}{ Tfh } & BCL6 & 0.160 & $* * * *$ & 0.134 & $* * * *$ & 0.117 & $*$ & 0.090 & 0.080 & 0.034 & 0.446 & 0.041 & 0.364 \\
\hline & IL2I & 0.198 & $* * * *$ & 0.070 & * & 0.246 & $* * * *$ & 0.227 & $* * * *$ & 0.120 & $* *$ & $0.08 I$ & $0.07 I$ \\
\hline \multirow[t]{2}{*}{ ThI7 } & STAT3 & 0.037 & 0.220 & 0.035 & 0.265 & 0.204 & $* * * *$ & 0.182 & $* * *$ & 0.035 & 0.423 & 0.058 & 0.200 \\
\hline & ILI7A & 0.122 & $* * * *$ & 0.004 & 0.892 & 0.132 & $* *$ & 0.121 & $*$ & 0.136 & $* *$ & 0.082 & 0.070 \\
\hline \multirow[t]{4}{*}{ Treg } & FOXP3 & 0.288 & $* * * *$ & 0.100 & $* *$ & 0.377 & $* * * *$ & 0.337 & $* * * *$ & 0.182 & $* * * *$ & 0.068 & 0.130 \\
\hline & CCR8 & 0.173 & $* * * *$ & 0.011 & $0.74 I$ & 0.368 & $* * * *$ & 0.338 & $* * * *$ & 0.224 & $* * * *$ & 0.122 & $* *$ \\
\hline & STAT5B & 0.304 & $* * * *$ & 0.260 & $* * * *$ & 0.289 & $* * * *$ & 0.272 & $* * * *$ & 0.292 & $* * * *$ & 0.264 & $* * * *$ \\
\hline & TGF- $\beta$ (TGFBI) & 0.371 & $* * * *$ & 0.216 & $* * * *$ & 0.097 & $*$ & 0.053 & 0.301 & 0.151 & $* * *$ & 0.085 & 0.059 \\
\hline \multirow[t]{5}{*}{$\mathrm{T}$ cell exhaustion } & PD-I(PDCDI) & 0.467 & $* * * *$ & 0.277 & $* * * *$ & 0.263 & $* * * *$ & 0.215 & $* * * *$ & 0.207 & $* * * *$ & 0.106 & $*$ \\
\hline & CTLA4 & 0.336 & $* * * *$ & 0.136 & $* * * *$ & 0.268 & $* * * *$ & 0.223 & $* * * *$ & 0.230 & $* * * *$ & 0.118 & $* *$ \\
\hline & LAG3 & 0.214 & $* * * *$ & 0.079 & $*$ & 0.220 & $* * * *$ & 0.192 & $* * *$ & 0.177 & $* * * *$ & 0.090 & $*$ \\
\hline & TIM-3(HAVCR2) & 0.218 & $* * * *$ & 0.021 & 0.514 & 0.190 & $* * * *$ & 0.150 & $* *$ & 0.264 & $* * * *$ & 0.173 & $* * *$ \\
\hline & GZMB & 0.392 & $* * * *$ & 0.208 & $* * * *$ & 0.141 & ** & 0.107 & $*$ & 0.121 & $* *$ & 0.027 & 0.546 \\
\hline
\end{tabular}

Notes: $* P<0.05$; $* * P<0.01 ; * * * P<0.001 ; * * * * P<0.0001$. None, correlation without adjustment; purity, correlation adjusted by purity.

Abbreviations: BRCA, breast cancer; STAD, stomach adenocarcinoma; LUAD, lung adenocarcinoma; TAM, tumor-associated macrophage; Th, T helper cell; Tfh, follicular helper T cell; Treg, regulatory T cell; Cor, R value of Spearman correlation.

CD8+T cells (marked by CD8A and CD8B), general $\mathrm{T}$ cells (marked by $\mathrm{CD} 3 \mathrm{D}$, CD3E, or $\mathrm{CD} 2$ ), B cells (marked by CD19 or CD79A), monocytes (marked by CD86 or CD115) had a positive relationship with DNASE1L3 expression in all these 9 cancers, except LIHC. TAM (marked by CCL2, CD86, or IL10) and neutrophils (marked by CD66b, CD11b, or CCR7) widely correlated with DNASE1L3 expression in these 9 cancers, except HNSC. As to M1/M2 macrophages, DNASE1L3 correlated with both in BRCA, SARC and UCEC, whereas with either M1 or M2 in the other 6 cancers. DNASE1L3 expression correlated with natural killer cells (marked by KIR2DL1, KIR2DL3, KR2DL4, KIR3DL1, KIR3DL2, KR3DL3, or KIR2DS4) in these 9 cancers, except KIRP and LIHC. DNASE1L3 expression correlated with DCs (marked by HLA-DPB1, HTHELA-DQB1, HLA-DRA, HLA-DPA1, BDCA-1, BDCA-4, or CD11c) in all 9 cancers except LIHC. In addition, DNASE1L3 expression correlated with Tregs (marked by FOXP3, CCR8, STAT5B, or TGF- $\beta$ ) and exhausted T cells (marked by PD-1, CTLA4, LAG3, TIM-3, or GZMB) in all these 9 cancers, except LIHC. Thus, DNASE1L3 expression universally correlation with immune cells in tumor microenvironment (TME), especially with DCs and T cells.
Then, the relationship between DNASE1L3 and DCs/ exhausted T cells in tumor and normal tissue was explored from TCGA through GEPIA (Table 2, Supplementary Table 3). Interestingly, DNASE1L3 generally correlated with DCs/exhausted T cells in these 9 cancers. In addition, CCR7 (a marker of neutrophil) strongly correlated with DNASE1L3 in most of these cancers. Accordingly, DNASE1L3 strongly correlated with CCL19 (the ligand of CCR7) in these cancers (Figure 5). Collectively, DNASE1L3 correlated with immune infiltrating cells in diverse cancers, which may participate in tumorigenesis and affect prognosis of cancer patients.

\section{DNASEIL3 is Downregulated in HCC, STAD, and LUAD}

DNASE1L3 expression in HCC, STAD, and LUAD was analyzed. Firstly, mRNA level was measured by qRT-PCR in HCC and adjacent normal tissues. DNASE1L3 was downregulated in HCC (Figure 6C). DNASE1L3, CCR7, and CCL19 were downregulated in STAD (Figure 6F-H). Then, DNASE1L3 expression in lung adenocarcinoma $(n=20)$ and HCC $(n=9)$ was detailed by immunohistochemistry using FFPE tissues. DNASE1L3 was downregulated in LUAD and HCC (Figure 6A-B, D-E). 
Table 2 Correlation Analysis Between DNASEIL3 and Immune Cells in Tumor/Normal Tissue of TCGA Through GEPIA2

\begin{tabular}{|c|c|c|c|c|c|c|c|c|c|c|c|c|c|}
\hline \multirow[t]{3}{*}{ Description } & \multirow[t]{3}{*}{ Gene Markers } & \multicolumn{4}{|c|}{ BRCA } & \multicolumn{4}{|c|}{ STAD } & \multicolumn{4}{|c|}{ LUAD } \\
\hline & & \multicolumn{2}{|c|}{ Tumor } & \multicolumn{2}{|c|}{ Normal } & \multicolumn{2}{|c|}{ Tumor } & \multicolumn{2}{|c|}{ Normal } & \multicolumn{2}{|c|}{ Tumor } & \multicolumn{2}{|c|}{ Normal } \\
\hline & & $\mathbf{R}$ & $P$ & $\mathbf{R}$ & $P$ & $\mathbf{R}$ & $P$ & $\mathbf{R}$ & $P$ & $\mathbf{R}$ & $P$ & $\mathbf{R}$ & $P$ \\
\hline \multirow[t]{7}{*}{ DCs } & HLA-DPBI & 0.520 & $* * * *$ & 0.180 & 0.054 & 0.390 & $* * * *$ & 0.280 & 0.100 & 0.380 & $* * * *$ & 0.086 & 0.510 \\
\hline & HLA-DQBI & 0.240 & $* * * *$ & 0.180 & 0.062 & 0.230 & $* * * *$ & 0.072 & 0.680 & 0.140 & $* *$ & 0.230 & 0.081 \\
\hline & HLA-DRA & 0.410 & $* * * *$ & 0.290 & $* *$ & 0.360 & $* * * *$ & 0.320 & 0.059 & 0.340 & $* * * *$ & 0.066 & 0.620 \\
\hline & HLA-DPAI & 0.440 & $* * * *$ & 0.250 & $* *$ & 0.370 & $* * * *$ & 0.330 & 0.053 & 0.330 & $* * * *$ & 0.230 & 0.078 \\
\hline & BDCA-I (CDIC) & 0.690 & $* * * *$ & 0.260 & $* *$ & 0.590 & $* * * *$ & 0.480 & $* *$ & 0.370 & $* * * *$ & 0.400 & $* *$ \\
\hline & BDCA4(NRPI) & 0.320 & $* * * *$ & 0.610 & $* * * *$ & 0.210 & $* * * *$ & 0.560 & $* * *$ & 0.042 & 0.360 & 0.440 & $* * *$ \\
\hline & CDIIc(ITGAX) & 0.300 & $* * * *$ & 0.083 & 0.380 & 0.300 & $* * * *$ & 0.200 & 0.250 & 0.270 & $* * * *$ & 0.061 & 0.640 \\
\hline \multirow[t]{5}{*}{$\mathrm{T}$ cell exhaustion } & PD-I(PDCDI) & 0.440 & $* * * *$ & 0.048 & 0.620 & 0.280 & $* * * *$ & 0.360 & $*$ & 0.170 & $* * *$ & 0.099 & 0.450 \\
\hline & CTLA4 & 0.310 & $* * * *$ & 0.033 & 0.730 & 0.300 & $* * * *$ & 0.460 & $* *$ & 0.220 & $* * * *$ & 0.150 & 0.270 \\
\hline & LAG3 & 0.170 & $* * * *$ & 0.150 & 0.120 & 0.240 & $* * * *$ & 0.072 & 0.670 & 0.140 & $* *$ & 0.074 & 0.580 \\
\hline & TIM-3(HAVCR2) & 0.210 & $* * * *$ & 0.420 & $* * * *$ & 0.270 & $* * * *$ & 0.170 & 0.330 & 0.270 & $* * * *$ & 0.180 & 0.170 \\
\hline & GZMB & 0.360 & $* * * *$ & 0.520 & $* * * *$ & 0.150 & $* *$ & 0.330 & $*$ & 0.077 & 0.089 & 0.031 & 0.820 \\
\hline
\end{tabular}

Notes: $* P<0.05$; $* * P<0.01$; $* * * P<0.001$; $* * * P<0.0001$. Tumor, correlation analysis between DNASEIL3 and immune cell markers in tumor tissue of TCGA through GEPIA2; normal, correlation analysis between DNASEIL3 and immune cell markers in normal tissue of TCGA through GEPIA2.

Abbreviations: BRCA, breast cancer; STAD, stomach adenocarcinoma; LUAD, lung adenocarcinoma; DCs, dendritic cells.

\section{Discussion}

In our present study, DNASE1L3 was markedly downregulated in various human cancers, and strongly correlated with poorer prognosis, especially in breast, kidney, liver cancer, stomach, lung adenocarcinoma and sarcoma. Furthermore, DNASE1L3 was related to immune infiltration, especially with CCR7/CCL19 axis. Our results suggest that DNASE1L3 could act as a prognostic marker and potential target for immune-related therapy in multiple human cancers.

DNASE1L3 (also termed DNase $\gamma$ ), an endonuclease, is a member of DNase I family. DNASE1L3, located on 3p14.3-p21.1, is recognized as a candidate tumor suppressor gene, ${ }^{11}$ since allelic loss of this region is common in cancers, including renal cell carcinoma and small cell lung cancers. ${ }^{37}$ Although a pathologic role of DNASE1L3 in cancer has yet to be extensively studied, DNASE1L3 might be involved in apoptosis. ${ }^{11,38,39}$ DNASE1L3 was overexpressed in the early stage of clear cell renal cancer, compared with late stage. ${ }^{40}$ In addition, DNASE1L3 was proposed as a prognostic factor of HCC. ${ }^{18}$ In our present study, DNASE1L3 had prognostic value in various types of cancers. Lower expression of DNASE1L3 correlated with poorer clinical outcomes in BRAD, STAD, LUAD, LIHC, ASRC, and KD; and probably in HNSC, melanoma, or UCEC. Moreover, DNASE1L3 correlated with immune infiltration and various immune cell markers in these cancers. Thus, our study provides supporting evidence about DNASE1L3 as a prognostic biomarker in cancers, as well as a potential target in immunotherapy of cancers.

\section{DNASEIL3 is Downregulated in Various Tumors and Correlated with Poor}

\section{Prognosis}

In our study, DNASE1L3 expression in cancer tissue was compared with adjacent normal tissue through independent datasets in Oncomine and TCGA. In Oncomine, DNASE1L3 was downregulated in the following cancers: breast, cervical, colorectal, esophageal, gastric, head and neck, kidney, liver, lung, ovarian, pancreatic, mesothelioma, leukemia, sarcoma, pleural malignant mesothelioma and pleural malignant fibrous histiocytoma in one or more datasets. By contrast, higher expression of DNASE1L3 was observed only with lymphoma in two datasets (Figure 1A). Lower expression of DANSE1L3 was observed in various types of human cancers in TCGA database through TIMER (Figure 1B). Thus, DNASE1L3 was generally downregulated in human cancers. Moreover, lower expression of DNASE1L3 correlated with poorer prognosis in kidney cancer (Figure 2K-L, Supplementary Figure 2L-M, Supplementary Figure 3K$\underline{\mathrm{M}}$ ), liver hepatocellular carcinoma (Figure 2M-N, Supplementary Figure 2E, Supplementary Figure 2N, 

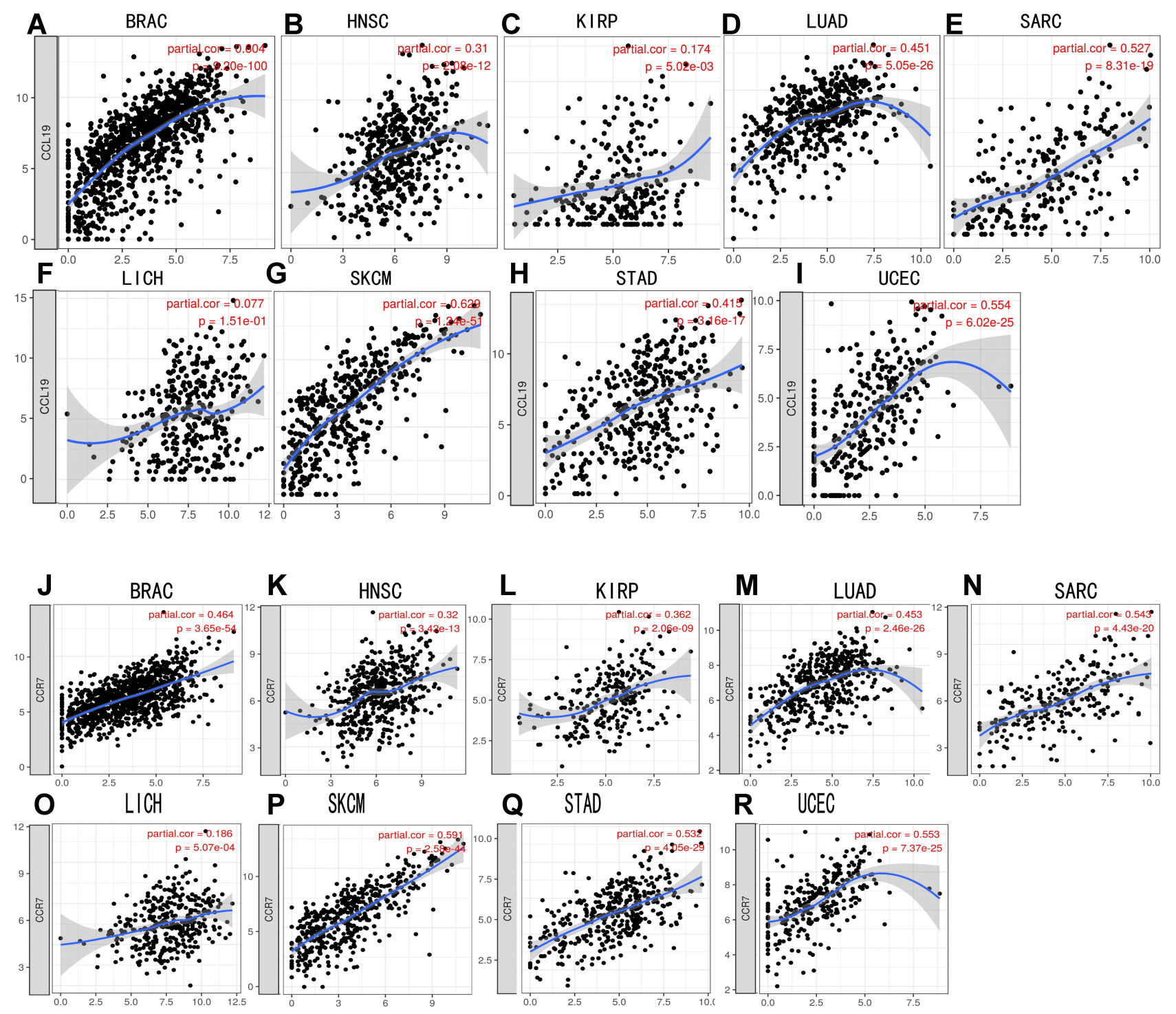

Figure 5 CCR7/CCLI9 were significantly correlated with the expression of DNASEIL3 in various cancers. (A-I) The correlation between DNASEIL3 expression and CCLI 9 in various cancers; (J-R) The correlation between DNASEIL3 expression and CCR7 in various cancers.

Abbreviations: BRCA, breast invasive carcinoma; HNSC, head and neck squamous cell carcinoma; KIRP, kidney renal clear cell carcinoma; LIHC, liver hepatocellular carcinoma; LUAD, lung adenocarcinoma; SARC, sarcoma; SKCM, skin cutaneous melanoma; STAD, stomach adenocarcinoma; UCEC, uterine carcinoma.

Supplementary Figure 3P), lung adenocarcinoma (Figure 2EF, Supplementary Figure 1M, Supplementary Figure 2O, Supplementary Figure 3Q), sarcoma (Figure 2O-P, Supplementary Figure 2U, Supplementary Figure 3Y) in PrognoScan, Kaplan-Meier Plotter, and TCGA databases. In addition, lower expression of DNASE1L3 correlated with poorer prognosis in breast cancer (Figure 2A-D, Supplementary Figure 2D, Supplementary Figure 2G, Supplementary Figure. 3C), HNSC (Supplementary Figure $1 \mathrm{~J}$, Supplementary Figure 2K, Supplementary Figure.3J), melanoma (Figure 2H, Supplementary Figure 3Z), STAD (Figure 2I-J, Supplementary Figure 2A, Supplementary Figure 2V, Supplementary Figure 3AA), and UCEC
(Supplementary Figure 2Z, Supplementary Figure 3AE) in PrognoScan and Kaplan-Meier Plotter databases, but not in TCGA. Then, prognostic value of DNASE1L3 in breast cancer was identified in bc-GenEcMiner V4.5, which specifically collects data from breast cancer studies and is widely used in breast cancer research. ${ }^{28,29}$ Accordingly, lower expression of DNASE1L3 in breast cancer correlated with poorer probability of OS and DFS (Figure 3A). Furthermore, DNASE1L3 was downregulated in HCC at both transcriptional and translational levels (Figure 6B, C, E), as well as in STAD and LUAD at translational level (Figure 6A, D, F). Collectively, DNASE1L3 may serve as a prognostic marker in breast cancer, kidney cancer, liver hepatocellular 

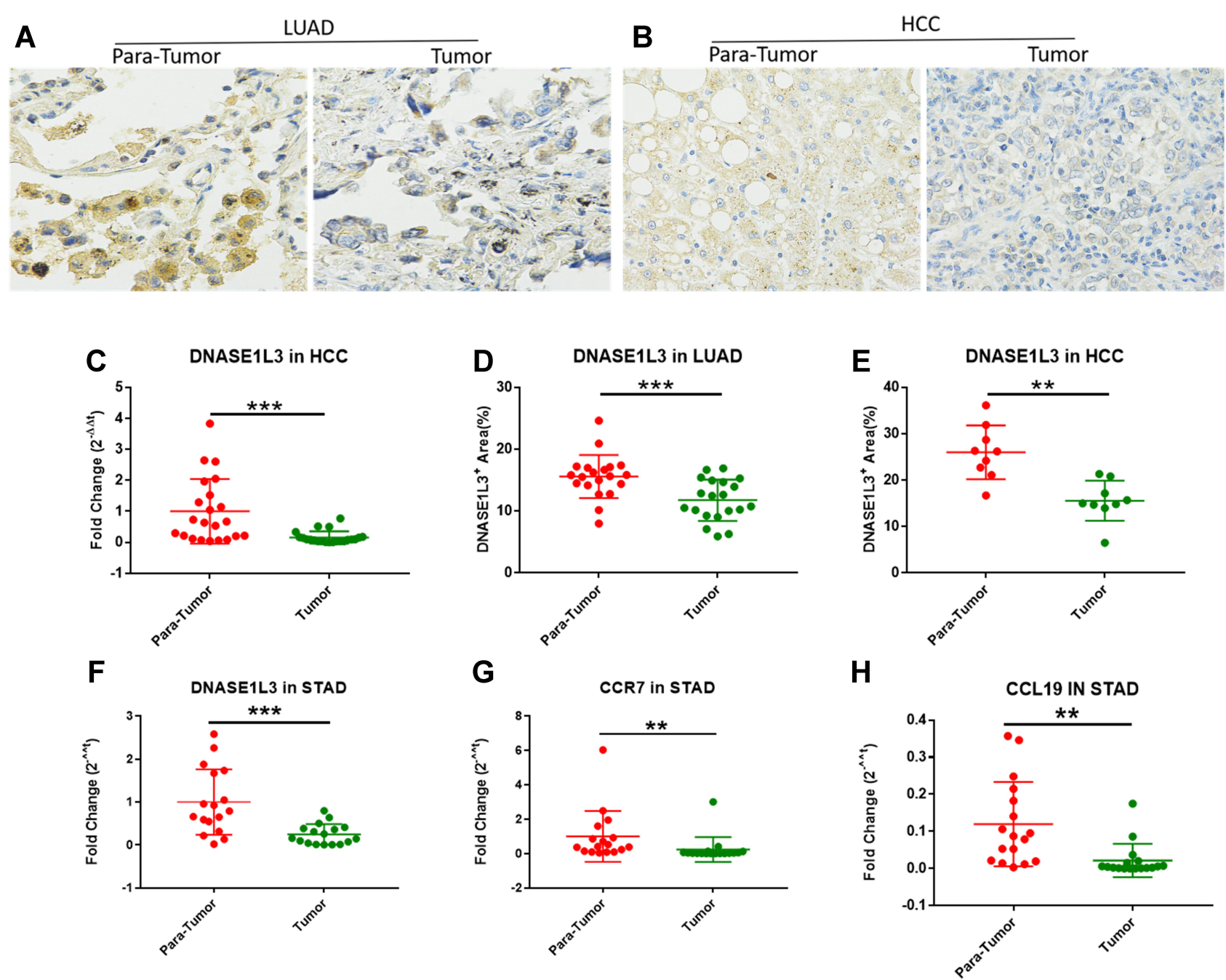

Figure 6 qRT-PCR results from HCC, stomach and lung adenocarcinoma cohorts. The expression of DNASEIL3 in LAUD and HCC were analyzed by immunohistochemical staining. The results indicated that DNASEIL3 was downregulated in LUAD compared with adjacent normal tissue (A, D) ( $n=20)$, and downregulated in HCC compared with adjacent normal tissue at protein level $(\mathbf{B}, \mathbf{E})(\mathrm{n}=9)$. qPCR was run to analyze the expression level of DNASEIL3, and the results showed that DNASEIL3 was downregulated in HCC compared with adjacent normal tissues $(\mathbf{C})(\mathrm{n}=22)$, and DNASEIL3, CCR7 and CCLI9 were downregulated in STAD compared with adjacent normal tissues $(\mathbf{F}-\mathbf{H})(\mathrm{n}=17)$. ${ }^{*} * \mathrm{P}<0.01$, $* * * P<0.001$.

Abbreviations: STAD, stomach adenocarcinoma; LUAD, lung adenocarcinoma; HCC, hepatocellular carcinoma; Para-Tumor, the paired adjacent tumor tissue.

carcinoma, lung adenocarcinoma and sarcoma. In addition, prognostic value of DNASE1L3 in HNSC, melanoma, STAD, and UCEC requires confirmation. As far as we know, this is the first report on prognostic value of DNASE1L3 in most of these types of cancers.

\section{DNASEIL3 Correlates with Immune Infiltration in Various Tumors}

Mutation of DNASE1L3 was frequently connected to autoimmune diseases, like systemic lupus erythematosus, rheumatoid arthritis, and type 1 diabetes. ${ }^{41,42}$ As previously reported, DNASE1L3 was involved in immune response related neutrophil activation, and might regulate acute inflammatory response and neutrophil mediated cytotoxicity. $^{21,22}$ To better understand functions of DNASE1L3 in cancers, we analyzed the relation between DNASE1L3 and immune infiltration in various cancers through TIMER. Interestingly, DNASE1L3 universally correlates with immune cells (Supplementary Figure 4). Specifically, DNASE1L3 correlates with prognosis in 9 cancers (breast invasive carcinoma, head and neck squamous cell carcinoma, kidney renal papillary cell carcinoma, liver hepatocellular carcinoma, lung adenocarcinoma, sarcoma, skin cutaneous melanoma, stomach adenocarcinoma, and uterine corpus endometrial carcinoma), and the strength of correlation varies from "moderate" $(0.40<|\mathrm{R}|<0.59)$ to "very week" $(0.00<|R|<0.19)$ (Figure 4). Thus, DNASE1L3 may 


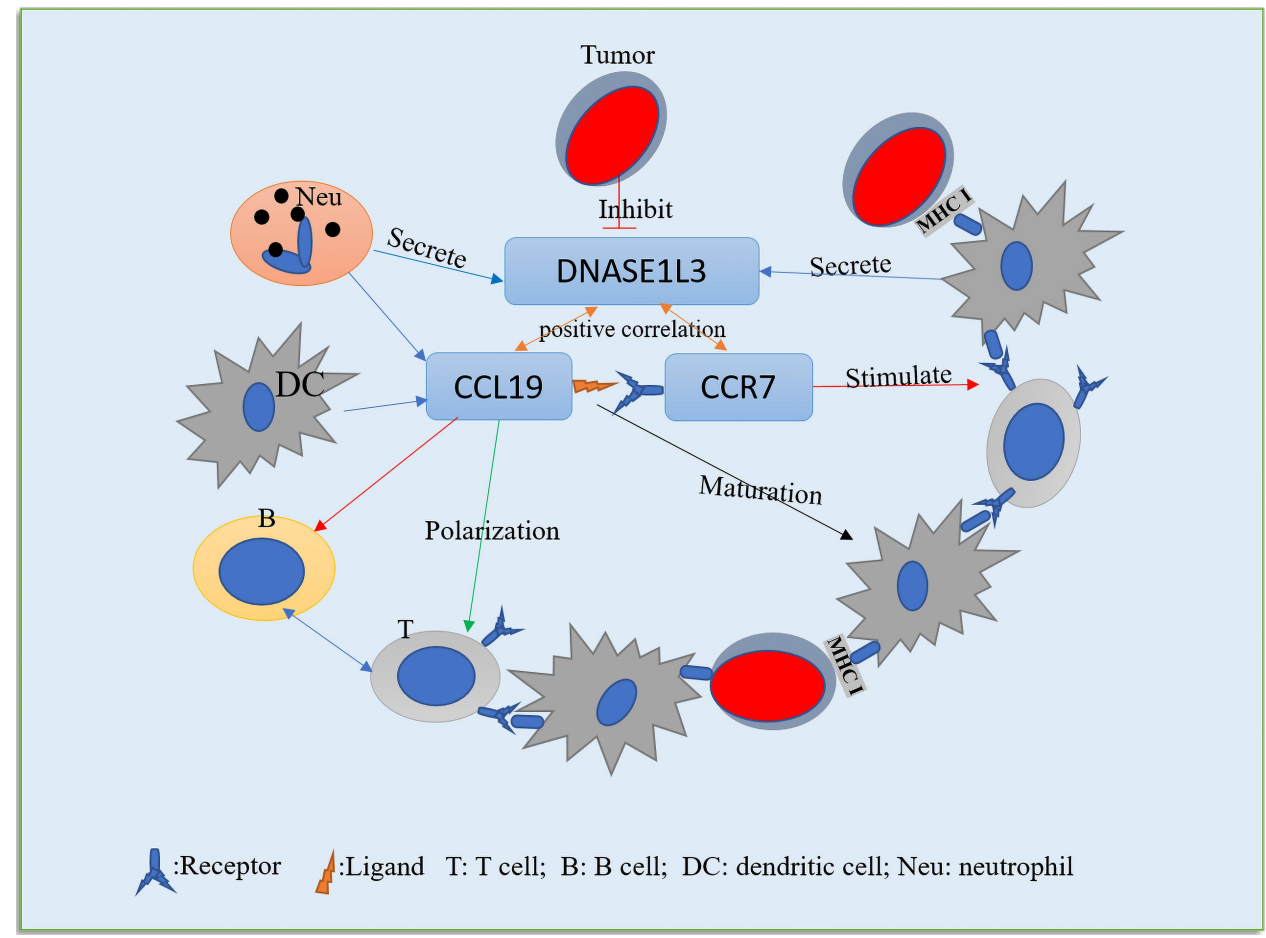

Figure 7 One probable underlying mechanism in the correlation among DNASEIL3, tumor cells and tumor immune microenvironment (TIM). The downregulation of DNASEIL3 in tumor, followed by the downregulation of CCR7/CCLI9 in TIM, which will affect the maturation of DC, the polarization of T cells, and the stimulation of B/T cells, will then affect the immune action on tumor cells, and finally lead to immune escape.

interact with immune infiltration in tumor microenvironment (TME), through which DNASE1L3 may affect prognosis of cancer patients. Moreover, DNASE1L3 correlates with multiple gene markers of tumor-infiltrating immune cells. For example, DNASE1L3 correlates with CD8+ T cell, general T cell and B cell in 8 of 9 cancers (except LIHC) (Table 1, Supplementary Table 2). DNASE1L3 universally correlates with monocytes, TAMs, M1/M2 macrophages, neutrophils, natural killer (NK) cells, dendritic cells (DCs), T-helper 1 (Th1) cells, T-helper 2 (Th2) cells, follicular helper T (Tfh) cells, T-helper 17 (Th17) cells, Tregs, and exhausted T cells, especially with CCR7 in neutrophils and BDCA-1 (CD1C) in dendritic cells. CCR7 (C-C motif chemokine receptor 7) is expressed in various lymphoid tissues, which activates $\mathrm{B}$ and $\mathrm{T}$ lymphocytes, as well as stimulating the maturation of dendritic cells. CCR7 acts in immune system and tumorigenesis when combined with its specific ligand CCL19 (C-C motif chemokine ligand 19). ${ }^{43-45}$ Accordingly, expression of DNASE1L3 significantly correlated with CCL19 (Figure 5). CCL19 promotes immune cells infiltrating into tumors to exert anti-tumor effects by inducing DCs into tumor tissue and by acting as a chemotactic factor on T cells through CCL19/CCR7 axis. ${ }^{46-48}$ Moreover, at least in lung adenocarcinoma and colorectal cancer cohorts, patients with CCL19-positive tumors had better prognosis. ${ }^{49,50}$ Combining findings from our present study and previous literature, we hypothesize a potential mechanism underlying the interaction of DNASE1L3 with tumor and TME (Figure 7): tumors could directly or indirectly inhibit CCL19-CCR7 axis by down-regulating DNASE1L3, which in turn produces a negative regulatory effect on the immune system, possibly by interfering with the maturation of DCs, inhibiting co-stimulatory activation of B/ $\mathrm{T}$ cells, and undermining infiltration of multiple immune cells, ultimately facilitating in immune escape. Collectively, DNASE1L3 is widely associated with immune infiltration in TME of various cancers, and CCR7/CCL19 axis may play a key role in this process. Further experiments should be designed to confirm this hypothesis and explore molecular mechanisms in detail.

\section{Conclusion}

In our study, we comprehensively analyzed expression profiling and prognostic value of DNASE1L3, as well as its correlation with the immune system in multiple cancers. Notably, DNASE1L3 is widely downregulated in 
human cancers and associated with poorer prognosis in various cancers, especially in breast cancer, kidney cancer, LIHC, LUAD, and SARC. In addition, DNASE1L3 expression is universally related to immune cell infiltration in TME of various cancers, especially with CCR7-CCL19 axis. Thus, we infer that tumor cells may cause decrease in immune infiltration through down-regulating DNASE1L3 expression, followed by suppressing CCR7/CCL19 axis. This may represent a new pathway in which tumor cells escape from immune surveillance. Demonstrating a pathologic relationship of DNASE1L3 down-regulation with immune infiltration in TME, and deciphering molecular mechanisms by which CCR7/CCL19 axis can modify anti-tumor response, will facilitate improvement in cancer immunotherapy.

\section{Data Sharing Statement}

Correspondence and requests for materials should be addressed to Jirun Peng at pengjr@medmail.com.cn or pengjr@bjsjth.cn.

\section{Acknowledgments}

We thank the Department of Pathology and the Central Lab of Beijing Shijitan Hospital, Capital Medical University, for providing technical support. This study was supported by National Natural Science Foundation of China (No. 82072601) and Beijing Natural Science Foundation (7182075).

\section{Disclosure}

All authors declare no conflicts of interest.

\section{References}

1. Role of immunity in therapy of human cancer. Discussion. $\mathrm{Br}$ J Cancer Suppl. 1973;1:299-308.

2. Sikora K, James N. Immune modulation and cancer. Br Med Bull. 1991;47(1):209-226.

3. Devaud C, John LB, Westwood JA, Darcy PK, Kershaw MH. Immune modulation of the tumor microenvironment for enhancing cancer immunotherapy. Oncoimmunology. 2013;2(8):e25961.

4. Sadreddini S, Baradaran B, Aghebati-Maleki A, et al. Immune checkpoint blockade opens a new way to cancer immunotherapy. $J$ Cell Physiol. 2019;234(6):8541-8549.

5. Brahmer JR, Tykodi SS, Chow LQM, et al. Safety and Activity of Anti-PD-L1 Antibody in Patients with Advanced Cancer. 2012;366 (26):2455-2465.

6. Chen L, Han X. Anti-PD-1/PD-L1 therapy of human cancer: past, present, and future. J Clin Invest. 2015;125(9):3384-3391.

7. Lin J, Yang X, Long J, et al. Pembrolizumab combined with lenvatinib as non-first-line therapy in patients with refractory biliary tract carcinoma. Hepatobiliary Surg Nutr. 2020;9(4):414-424.
8. Zhang H, Liu H, Shen Z, et al. Tumor-infiltrating neutrophils is prognostic and predictive for postoperative adjuvant chemotherapy benefit in patients with gastric cancer. Ann Surg. 2018;267 (2):311-318.

9. Waniczek D, Lorenc Z, Snietura M, Wesecki M, Kopec A, TumorAssociated Macrophages M-WM. Regulatory T cells infiltration and the clinical outcome in colorectal cancer. Arch Immunol Ther Exp (Warsz). 2017;65(5):445-454.

10. Kumpers C, Jokic M, Haase O, et al. Immune Cell Infiltration of the Primary Tumor, Not PD-L1 status, is associated with improved response to checkpoint inhibition in metastatic melanoma. Front Med (Lausanne). 2019;6:27.

11. Rodriguez AM, Rodin D, Nomura H, Morton CC, Weremowicz S, Schneider MC. Identification, localization, and expression of two novel human genes similar to deoxyribonuclease I. Genomics. 1997;42(3):507-513.

12. Shiokawa D, Tanuma S. Characterization of human DNase I family endonucleases and activation of DNase gamma during apoptosis. Biochemistry. 2001;40(1):143-152.

13. Shiokawa D, Ohyama H, Yamada T, Takahashi K, Tanuma S. Identification of an endonuclease responsible for apoptosis in rat thymocytes. Eur J Biochem. 1994;226(1):23-30.

14. Liu QY, Pandey S, Singh RK, et al. DNaseY: a rat DNasel-like gene coding for a constitutively expressed chromatin-bound endonuclease. Biochemistry. 1998;37(28):10134-10143.

15. Errami Y, Naura AS, Kim H, et al. Apoptotic DNA fragmentation may be a cooperative activity between caspase-activated deoxyribonuclease and the poly(ADP-ribose) polymerase-regulated DNAS1L3, an endoplasmic reticulum-localized endonuclease that translocates to the nucleus during apoptosis. J Biol Chem. 2013;288 (5):3460-3468.

16. Serpas L, Chan RWY, Jiang P, et al. Dnase113 deletion causes aberrations in length and end-motif frequencies in plasma DNA. Proc Natl Acad Sci U S A. 2019;116(2):641-649.

17. Sjoblom T, Jones $\mathrm{S}$, Wood LD, et al. The consensus coding sequences of human breast and colorectal cancers. Science. 2006;314 (5797):268-274.

18. Xu B, Lv W, Li X, Zhang L, Lin J. Prognostic genes of hepatocellular carcinoma based on gene coexpression network analysis. J Cell Biochem. 2019.

19. Sisirak V, Sally B, D'Agati V, et al. Digestion of chromatin in apoptotic cell microparticles prevents autoimmunity. Cell. 2016;166 (1):88-101.

20. Liu QY, Ribecco M, Pandey S, Walker PR, Sikorska M. Apoptosisrelated functional features of the DNaseI-like family of nucleases. Ann N Y Acad Sci. 1999;887:60-76.

21. Jimenez-Alcazar M, Rangaswamy C, Panda R, et al. Host DNases prevent vascular occlusion by neutrophil extracellular traps. Science. 2017;358(6367):1202-1206.

22. Xie X, Liu PS, Percipalle P. Analysis of Global Transcriptome Change in Mouse Embryonic Fibroblasts After dsDNA and dsRNA Viral Mimic Stimulation. Front Immunol. 2019;10:836.

23. Tang Z, Kang B, Li C, Chen T, Zhang Z. GEPIA2: an enhanced web server for large-scale expression profiling and interactive analysis. Nucleic Acids Res. 2019.

24. Rhodes DR, Kalyana-Sundaram S, Mahavisno V, et al. Oncomine 3.0: genes, pathways, and networks in a collection of 18,000 cancer gene expression profiles. Neoplasia. 2007;9(2):166-180.

25. Mizuno H, Kitada K, Nakai K, Sarai A. PrognoScan: a new database for meta-analysis of the prognostic value of genes. BMC Med Genomics. 2009;2:18.

26. Lanczky A, Nagy A, Bottai G, et al. miRpower: a web-tool to validate survival-associated miRNAs utilizing expression data from 2178 breast cancer patients. Breast Cancer Res Treat. 2016;160 (3):439-446. 
27. Nagy A, Lanczky A, Menyhart O, Gyorffy B. Validation of miRNA prognostic power in hepatocellular carcinoma using expression data of independent datasets. Sci Rep. 2018;8(1):9227.

28. Jezequel P, Campone M, Gouraud W, et al. bc-GenExMiner: an easy-to-use online platform for gene prognostic analyses in breast cancer. Breast Cancer Res Treat. 2012;131(3):765-775.

29. Jezequel P, Frenel JS, Campion L, et al. bc-GenExMiner 3.0: new mining module computes breast cancer gene expression correlation analyses. Database (Oxford). 2013;2013:bas060.

30. Li T, Fan J, Wang B, et al. TIMER: a web server for comprehensive analysis of tumor-infiltrating immune cells. Cancer Res. 2017;77(21): e108-e110.

31. Li B, Severson E, Pignon JC, et al. Comprehensive analyses of tumor immunity: implications for cancer immunotherapy. Genome Biol. 2016;17(1):174.

32. Aran D, Sirota M, Butte AJ. Systematic pan-cancer analysis of tumour purity. Nat Commun. 2015;6:8971.

33. Pan JH, Zhou H, Cooper L, et al. LAYN is a prognostic biomarker and correlated with immune infiltrates in gastric and colon cancers Front Immunol. 2019;10:6.

34. Siemers NO, Holloway JL, Chang H, et al. Genome-wide association analysis identifies genetic correlates of immune infiltrates in solid tumors. PLoS One. 2017;12(7):e0179726.

35. Danaher P, Warren S, Dennis L, et al. Gene expression markers of Tumor Infiltrating Leukocytes. J Immunother Cancer. 2017;5:18

36. Sousa S, Maatta J. The role of tumour-associated macrophages in bone metastasis. J Bone Oncol. 2016;5(3):135-138.

37. Kovacs G, Erlandsson R, Boldog F, et al. Consistent chromosome 3p deletion and loss of heterozygosity in renal cell carcinoma. Proc Natl Acad Sci U S A. 1988;85(5):1571-1575.

38. Yakovlev AG, Wang G, Stoica BA, Simbulan-Rosenthal CM, Yoshihara K, Smulson ME. Role of DNAS1L3 in Ca2+- and Mg2 +-dependent cleavage of DNA into oligonucleosomal and high molecular mass fragments. Nucleic Acids Res. 1999;27(9):1999-2005.

39. Mizuta R, Araki S, Furukawa M, et al. DNase gamma is the effector endonuclease for internucleosomal DNA fragmentation in necrosis. PLoS One. 2013;8(12):e80223.

40. Bhalla S, Chaudhary K, Kumar R, et al. Gene expression-based biomarkers for discriminating early and late stage of clear cell renal cancer. Sci Rep. 2017;7:44997.
41. Almlof JC, Nystedt S, Leonard D, et al. Whole-genome sequencing identifies complex contributions to genetic risk by variants in genes causing monogenic systemic lupus erythematosus. Hum Genet. 2019;138(2):141-150.

42. Westra HJ, Martinez-Bonet M, Onengut-Gumuscu S, et al. Finemapping and functional studies highlight potential causal variants for rheumatoid arthritis and type 1 diabetes. Nat Genet. 2018;50 (10):1366-1374

43. Diao R, Wei W, Zhao J, Tian F, Cai X, Duan YG. CCL19/CCR7 contributes to the pathogenesis of endometriosis via PI3K/Akt pathway by regulating the proliferation and invasion of ESCs. Am J Reprod Immunol. 2017;78:5.

44. Cheng S, Guo J, Yang Q, Yang X. Crk-like adapter protein regulates CCL19/CCR7-mediated epithelial-to-mesenchymal transition via ERK signaling pathway in epithelial ovarian carcinomas. Med Oncol. 2015;32(3):47.

45. Portero-Sainz I. Gomez-Garcia de Soria V, Cuesta-Mateos C, et al. A high migratory capacity of donor T-cells in response to the lymph node homing receptor CCR7 increases the incidence and severity of GvHD. Bone Marrow Transplant. 2017;52(5):745-752.

46. Braun SE, Chen K, Foster RG, et al. The CC chemokine CK beta-11/ MIP-3 beta/ELC/Exodus 3 mediates tumor rejection of murine breast cancer cells through NK cells. J Immunol. 2000;164(8):4025-4031.

47. Hillinger S, Yang SC, Zhu L, et al. EBV-induced molecule 1 ligand chemokine (ELC/CCL19) promotes IFN-gamma-dependent antitumor responses in a lung cancer model. J Immunol. 2003;171 (12):6457-6465.

48. Hillinger S, Yang SC, Batra RK, et al. CCL19 reduces tumour burden in a model of advanced lung cancer. Br J Cancer. 2006;94 (7):1029-1034

49. Lu J, Zhao J, Feng H, et al. Antitumor efficacy of CC motif chemokine ligand 19 in colorectal cancer. Dig Dis Sci. 2014;59 (9):2153-2162.

50. Itakura M, Terashima Y, Shingyoji M, et al. High CC chemokine receptor 7 expression improves postoperative prognosis of lung adenocarcinoma patients. Br J Cancer. 2013;109(5):1100-1108.
OncoTargets and Therapy

\section{Publish your work in this journal}

OncoTargets and Therapy is an international, peer-reviewed, open access journal focusing on the pathological basis of all cancers, potential targets for therapy and treatment protocols employed to improve the management of cancer patients. The journal also focuses on the impact of management programs and new therapeutic agents and protocols on patient perspectives such as quality of life, adherence and satisfaction. The manuscript management system is completely online and includes a very quick and fair peer-review system, which is all easy to use. Visit http://www.dovepress.com/ testimonials.php to read real quotes from published authors. 